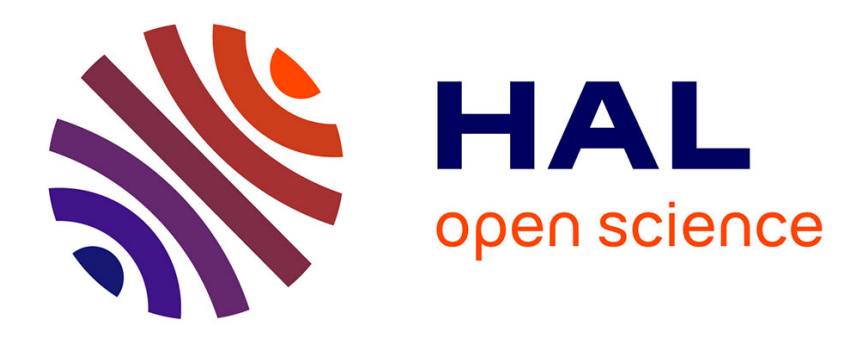

\title{
On the nonlinear normal modes of free vibration of piecewise linear systems
}

B.V. Uspensky, Konstantin Avramov

\section{To cite this version:}

B.V. Uspensky, Konstantin Avramov. On the nonlinear normal modes of free vibration of piecewise linear systems. Journal of Sound and Vibration, 2014, 333 (14), pp. 3252-3265. 10.1016/j.jsv.2014.02.039 . hal-01354036

\section{HAL Id: hal-01354036 \\ https://hal.science/hal-01354036}

Submitted on 17 Aug 2016

HAL is a multi-disciplinary open access archive for the deposit and dissemination of scientific research documents, whether they are published or not. The documents may come from teaching and research institutions in France or abroad, or from public or private research centers.
L'archive ouverte pluridisciplinaire HAL, est destinée au dépôt et à la diffusion de documents scientifiques de niveau recherche, publiés ou non, émanant des établissements d'enseignement et de recherche français ou étrangers, des laboratoires publics ou privés.

\section{(c)(1)}

Distributed under a Creative Commons Attribution| 4.0 International License 


\title{
On the nonlinear normal modes of free vibration of piecewise linear systems
}

\author{
B.V. Uspensky ${ }^{\mathrm{b}}$, K.V. Avramov ${ }^{\mathrm{a}, \mathrm{b}}$ \\ a Podgorny Institute for Mechanical Engineering, National Academy of Science of Ukraine, Department of Vibrations, \\ 2/10 Dm. Pozharskogo St., 61046 Kharkiv, Ukraine \\ b Department of Gas and Fluid Mechanics, National Technical University “KhPI”, Frunze St. 21, Kharkiv 61002, Ukraine
}

\begin{abstract}
A modification of the Shaw-Pierre nonlinear normal modes is suggested in order to analyze the vibrations of a piecewise linear mechanical systems with finite degrees of freedom. The use of this approach allows one to reduce to twice the dimension of the nonlinear algebraic equations system for nonlinear normal modes calculations in comparison with systems obtained by previous researchers. Two degrees of freedom and fifteen degrees of freedom nonlinear dynamical systems are investigated numerically by using nonlinear normal modes.
\end{abstract}

\section{Introduction}

Finite degree of freedom dynamical systems with piecewise linear elastic forces can be used to describe the vibrations of a wide class of machines and mechanisms. Therefore many efforts have been made to analyze the dynamics of such systems [1-5]. Free, forced and parametric vibrations of mechanical systems have been investigated by the Shaw-Pierre nonlinear normal modes [6]. The nonlinear normal modes of piecewise linear systems have been treated by Chen, Shaw [7] and Jiang, Pierre, Shaw [8]. The general theory of Shaw-Pierre nonlinear normal modes is considered in $[9,10]$. Theoretical investigations of nonlinear normal modes have been reviewed by Mikhlin and Avramov [6] and applications of nonlinear normal modes for engineering have been reviewed by Avramov and Mikhlin [11].

An approach for Shaw-Pierre nonlinear normal modes calculations is suggested for the analysis of piecewise linear systems in this paper. As a result of the use of this approach the dimension of the nonlinear algebraic equations system for the nonlinear normal mode is half that of the dimension of the system obtained by Jiang, Pierre and Shaw [8]. The torsional vibrations of two degrees of freedom and fifteen degrees of freedom systems with piecewise linear elastic moments are studied using this suggested approach. Properties of the nonlinear normal modes of the torsional vibrations are analyzed.

\section{Problem formulation}

A finite degree of freedom mechanical system with trilinear elastic forces is considered in the following matrix form:

$$
\mathbf{M} \ddot{\mathbf{z}}+\mathbf{F}(\mathbf{z})=0
$$




$$
\mathbf{F}(\mathbf{z})=\left\{\begin{array}{l}
\mathbf{K}_{\mathbf{1}} \mathbf{z} ; \quad \Delta_{2} \leq \mathbf{h}^{\mathbf{T}} \mathbf{z} \leq \Delta_{3} \\
\mathbf{K}_{\mathbf{2}} \mathbf{z}-\mathbf{b}_{\mathbf{2}} ; \mathbf{h}^{\mathbf{T}} \mathbf{z}<\Delta_{2} ; \\
\mathbf{K}_{\mathbf{3}} \mathbf{z}-\mathbf{b}_{\mathbf{3}} ; \Delta_{3}<\mathbf{h}^{\mathbf{T}} \mathbf{z}
\end{array}\right.
$$

where $\mathbf{z}$ is an $N$ dimensional vector of the generalized coordinates; $\mathbf{M}=\operatorname{diag}\left(M_{1}, \ldots, M_{N}\right)$; $\mathbf{h}$ is an $N$ dimensional vector; $\mathbf{K}_{\mathbf{1}}, \mathbf{K}_{2}, \mathbf{K}_{\mathbf{3}}$ are $N \times N$ dimensional matrixes; and the vectors $\mathbf{b}_{\mathbf{2}}, \mathbf{b}_{\mathbf{3}}$ afford continuity of the trilinear elastic forces; $\Delta_{2}<0, \Delta_{3}>0$.

Thus, a general case for finite degree of freedom mechanical systems with trilinear elastic forces is considered.

System (1) is transformed into the principal coordinates $\eta$ of the system in the linear region $\Delta_{2} \leq \mathbf{h}^{\mathbf{T}} \mathbf{z} \leq \Delta_{3}$, and these are the same principal coordinates as used in $[7,8]$. The following change of variables is used:

$$
\mathbf{z}=\mathbf{Q} \boldsymbol{\eta}
$$

where matrix $\mathbf{Q}$ consists of the eigenvectors of the matrix $\mathbf{M}^{-\mathbf{1}} \mathbf{K}_{\mathbf{1}}$. Therefore system (1) takes the following form with respect to the vector $\boldsymbol{\eta}$ :

$$
\ddot{\boldsymbol{\eta}}+\boldsymbol{\Lambda}_{\mathbf{1}} \boldsymbol{\eta}=\mathbf{f}(\boldsymbol{\eta}),
$$

where $\boldsymbol{\Lambda}_{\mathbf{1}}=\mathbf{Q}^{-\mathbf{1}} \mathbf{M}^{-\mathbf{1}} \mathbf{K}_{\mathbf{1}} \mathbf{Q}=\operatorname{diag}\left(\omega_{1}^{2}, \ldots, \omega_{N}^{2}\right)$ is a diagonal matrix; and $\omega_{1}, \ldots, \omega_{N}$ are the eigenfrequencies of the system in the linear region. The piecewise linear-vector function $\mathbf{f}(\boldsymbol{\eta})=\left(f_{1}, \ldots, f_{N}\right)$ takes the form:

$$
\mathbf{f}(\boldsymbol{\eta})=\left\{\begin{array}{c}
0, \Delta_{2} \leq \mathbf{h}^{\mathbf{T}} \mathbf{Q} \boldsymbol{\eta} \leq \Delta_{3} ; \\
\mathbf{Z}^{(2)} \boldsymbol{\eta}+\mathbf{Q}^{-\mathbf{1}} \mathbf{M}^{-\mathbf{1}} \mathbf{b}_{\mathbf{2}}, \mathbf{h}^{\mathrm{T}} \mathbf{Q} \boldsymbol{\eta}<\Delta_{2} ; \\
\mathbf{Z}^{(3)} \boldsymbol{\eta}+\mathbf{Q}^{-\mathbf{1}} \mathbf{M}^{-\mathbf{1}} \mathbf{b}_{\mathbf{3}}, \quad \Delta_{3}<\mathbf{h}^{\mathrm{T}} \mathbf{Q} \boldsymbol{\eta},
\end{array}\right.
$$

where $\mathbf{Z}^{(2)}=\mathbf{\Lambda}_{\mathbf{1}}-\mathbf{Q}^{-\mathbf{1}} \mathbf{M}^{-\mathbf{1}} \mathbf{K}_{\mathbf{2}} \mathbf{Q} ; \mathbf{Z}^{(3)}=\boldsymbol{\Lambda}_{\mathbf{1}}-\mathbf{Q}^{-\mathbf{1}} \mathbf{M}^{-\mathbf{1}} \mathbf{K}_{\mathbf{3}} \mathbf{Q} ; \mathbf{Z}^{(2)}=\left\{z_{i j}^{(2)}\right\} ; \mathbf{Z}^{(3)}=\left\{z_{i j}^{(3)}\right\}$.

\section{Calculations of the Shaw-Pierre nonlinear normal modes}

All the generalized coordinates and their velocities are separated into master coordinates and slave coordinates $[9,10]$. The variables $\left(\eta_{i}, \dot{\eta}_{i}\right)$ are chosen as master coordinates. Other variables $\left(\eta_{1}, \dot{\eta}_{1}, \ldots, \eta_{i-1}, \dot{\eta}_{i-1}, \eta_{i+1}, \dot{\eta}_{i+1}, \ldots, \dot{\eta}_{N}\right)$ are defined as slave coordinates. The master coordinates $\left(\eta_{i}, \dot{\eta}_{i}\right)$ satisfy the change of variables:

$$
\eta_{i}(t)=a(t) \cos \varphi(t) ; \dot{\eta}_{i}(t)=-\omega_{i} a(t) \cos \varphi(t) .
$$

Then the slave coordinates are obtained from the equations of the nonlinear normal modes:

$$
\begin{gathered}
\eta_{j}=P_{j}(a, \varphi) ; \\
\dot{\eta}_{j}=R_{j}(a, \varphi) ; j=1, \ldots, i-1, i+1, \ldots, N .
\end{gathered}
$$

Pesheck, Pierre, and Shaw [12-14] calculated the functions $P_{j}(a, \varphi), R_{j}(a, \varphi)$ to obtain the nonlinear normal modes. It is shown in this paper that it is enough to calculate the functions $P_{j}(a, \varphi)$ to determine the nonlinear normal modes. Thus, the functions $R_{j}(a, \varphi)$ are not considered.

The motions at the nonlinear normal mode are described by the following dynamical system [8]:

$$
\begin{aligned}
& \dot{a}=-\frac{f_{i}}{\omega_{i}} \sin \varphi ; \\
& \dot{\varphi}=\omega_{i}-\frac{f_{i}}{a \omega_{i}} \cos \varphi .
\end{aligned}
$$

The functions $P_{j}(a, \varphi)$ are expanded using the orthonormal trial functions $T_{l, m}$ :

$$
P_{j}(a, \varphi)=\sum_{l=1}^{N_{a}} \sum_{m=1}^{N_{\varphi}} C_{j}^{(l, m)} T_{l, m}(a, \varphi),
$$

where $T_{l, m}(a, \varphi)=A_{l}(a) \cos (m-1) \varphi ; A_{l}(a), l=1, \ldots, N_{a}$ are orthonormal trial functions on the interval $\left[a_{1,0} ; a_{2,0}\right] ;$ and $C_{j}^{(l, m)}$ are unknown coefficients which must be calculated. We stress that the expansions of the functions $R_{j}(a, \varphi)$ from (7) are used in [8,9], so here these expansions are not considered. This reduces the dimension of the nonlinear algebraic equation system by twice, in comparison with the approach suggested in [8,9]. This is the main advantage of the suggested method.

It can be shown from Eqs. (7) and (9) that:

$$
\begin{gathered}
\dot{\eta}_{j}=\sum_{l=1}^{N_{a}} \sum_{m=1}^{N_{\varphi}}\left(A_{l}^{\prime} \cos [(m-1) \varphi] \cdot \dot{a}-(m-1) A_{l} \sin [(m-1) \varphi] \cdot \dot{\varphi}\right) ; \\
\ddot{\eta}_{j}=\sum_{l=1 m=1}^{N_{a}} \sum_{m=1}^{N_{\varphi}}\left(\cos [(m-1) \varphi]\left[A_{l}^{\prime \prime} \dot{a}^{2}+A_{l}^{\prime} \ddot{a}-(m-1)^{2} A_{l} \dot{\varphi}^{2}\right]-\sin [(m-1) \varphi]\left[2(m-1) A_{l}^{\prime} \dot{a} \dot{\varphi}+A_{l} \ddot{\varphi}\right]\right) .
\end{gathered}
$$


Eq. (8) is substituted into (10) to calculate the first derivatives.

The second derivatives $\ddot{a}, \ddot{\varphi}$ take the following form:

$$
\begin{gathered}
\ddot{a}=\frac{d}{\mathrm{~d} t}\left(-\frac{f_{i}}{\omega_{i}} \sin \varphi\right)=-\frac{1}{\omega_{i}}\left(\left[\frac{\partial f_{i}}{\partial a} \dot{a}+\dot{\varphi} \frac{\partial f_{i}}{\partial \varphi}\right] \sin \varphi+f_{i} \cos \varphi\right) ; \\
\ddot{\varphi}=\frac{d}{\mathrm{~d} t}\left(\omega_{i}-\frac{f_{i}}{a \omega_{i}} \cos \varphi\right)=-\frac{1}{\omega_{i} a}\left(\left[\frac{\partial f_{i}}{\partial a} \dot{a}+\frac{\partial f_{i}}{\partial \phi} \dot{\varphi}-f_{i} \frac{\dot{a}}{a}\right] \cos \varphi-f_{i} \sin \varphi \cdot \dot{\varphi}\right) .
\end{gathered}
$$

Note that the partial derivatives of the function $f_{i}$ are calculated as

$$
\begin{gathered}
\frac{\partial f_{i}}{\partial a}=\left\{\begin{array}{cr}
0 ; & \Delta_{2} \leq h^{T} Q \eta \leq \Delta_{3} ; \\
\sum_{k=1}^{N} z_{i k}^{(2)} \frac{\partial \eta_{k}}{\partial a} ; & h^{T} Q \eta<\Delta_{2} ; \\
\sum_{k=1}^{N} z_{i k}^{(3)} \frac{\partial \eta_{k} ;}{\partial a} & \Delta_{3}<h^{T} Q \eta ;
\end{array}\right. \\
\frac{\partial f_{i}}{\partial \varphi}=\left\{\begin{array}{cr}
0 ; & \Delta_{2} \leq h^{T} Q \eta \leq \Delta_{3} ; \\
\sum_{k=1}^{N} z_{i k}^{(2)} \frac{\partial \eta_{k}}{\partial \varphi} ; & h^{T} Q \eta<\Delta_{2} ; \\
\sum_{k=1}^{N} z_{i k}^{(3)} \frac{\partial \eta_{k}}{\partial \varphi} ; & \Delta_{3}<h^{T} Q \eta
\end{array}\right.
\end{gathered}
$$

where

$$
\begin{gathered}
\frac{\partial \eta_{k}}{\partial a}=\left\{\begin{array}{c}
\sum_{l=1}^{N_{a}} \sum_{m=1}^{N_{\varphi}} C_{k}^{l, m} A_{l}^{\prime} \cos [(m-1) \varphi] ; k \neq i ; \\
\cos \phi ; k=i ;
\end{array}\right. \\
\frac{\partial \eta_{k}}{\partial \varphi}=\left\{\begin{array}{c}
-\sum_{l=1}^{N_{a}} \sum_{m=1}^{N_{\varphi}} C_{k}^{l, m}(m-1) A_{l} \sin [(m-1) \varphi] ; k \neq i ; \\
-a \sin \varphi ; k=i .
\end{array}\right.
\end{gathered}
$$

The coefficients $C_{j}^{(l, m)}$ are the main unknowns of the nonlinear normal mode (9). In order to obtain these coefficients the Galerkin method is applied to the dynamical system (4)

$$
\begin{gathered}
\int_{a_{1,0}}^{a_{2,0}} \int_{0}^{2 \pi}\left\{\ddot{\eta}_{j}+\omega_{j}{ }^{2} \eta_{j}-f_{j}(\eta)\right\} T_{l, m} \mathrm{~d} a \mathrm{~d} \varphi=0 \\
j=1, \ldots, N ; j \neq i \\
l=1, \ldots, N_{a} ; m=1, \ldots, N_{\varphi} .
\end{gathered}
$$

Eqs. (10)-(12) are substituted into Eq. (14), and as a result the system of $(N-1) N_{a} N_{\varphi}$ nonlinear algebraic equations with respect to the coefficients $C_{j}^{l, m}$ is obtained. When these coefficients have been calculated the nonlinear normal modes (9) are determined. If the nonlinear normal modes are calculated the dynamics for these modes are described by system (8).

The Newton method is used for the numerical solution of the nonlinear algebraic equation system. Integration (14) is performed over a region $a \in\left[0 ; a_{0}\right], \varphi \in[0 ; 2 \pi]$, and the region of the integration $a \in\left[0 ; a_{0}\right]$ is divided into $N_{s}$ sections $\left\lfloor a_{j} ; a_{j+1}\right\rfloor ; j=1, \ldots, N_{s} ; a_{1}=0 ; a_{N_{s}+1}=a_{0}$. The orthonormal trial functions $A_{l}, l=1, \ldots, N_{a}$ are used for each section. Linear trial functions are used for every section $[8,9]$ :

$$
A_{1}(a)=\frac{a-a_{j}}{a_{j+1}-a_{j}} ; A_{2}=\frac{a_{j+1}-a}{a_{j+1}-a_{j}}
$$

\section{Nonlinear normal modes for torsional vibrations}

\subsection{System with fifteen degrees of freedom}

Analysis of the models with many degrees of freedom is one of the most important applications of nonlinear normal modes. Shaw and Pierre [12-14] were the first to analyze this problem. They used the finite element method to discretize a nonlinear continuous system. A detailed review of these investigations is published in [11], and one more application of nonlinear normal modes is presented in this paper.

A boxer internal combustion engine power transmission is considered. The main elements of the power transmission are shown in Fig. 1. It consists of two crankshafts and a gear train, with each crankshaft having three cranks. These crankshafts 


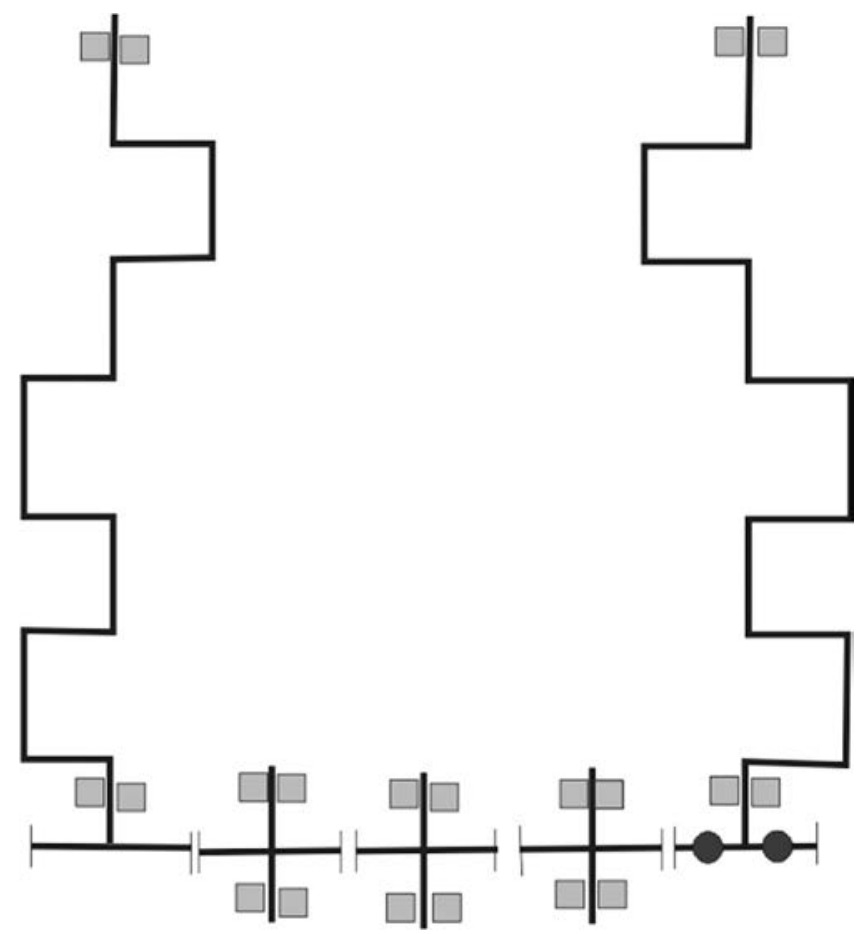

Fig. 1. Sketch of the power transmission.

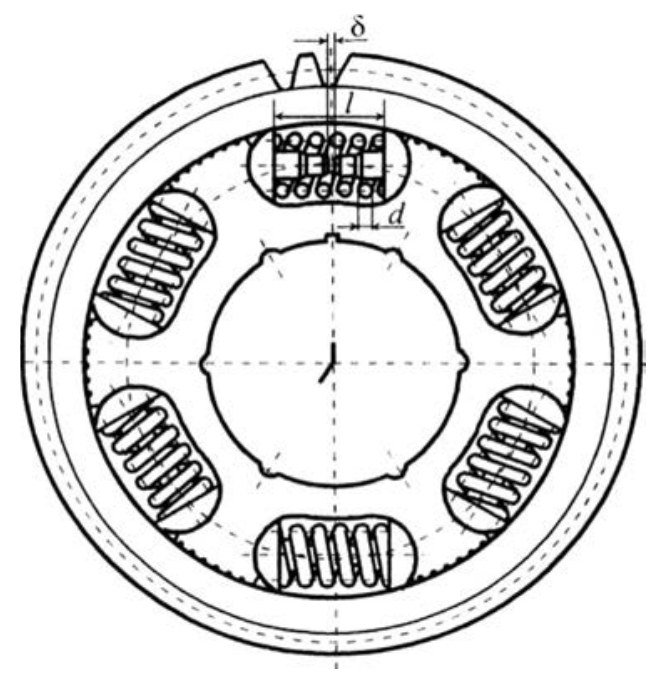

Fig. 2. Sketch of the clutch.

are connected by a gear train comprising five spur gears (Fig. 1). A clutch is installed into one gear as denoted by two black circles in Fig. 1. A sketch of this clutch is shown in Fig. 2. The clutch consists of two half clutches with sectors. Two preloaded springs are installed within each sector.

All internal combustion engines are powerful sources of torsional vibrations. Time-periodic moments act on each crank of the shaft [15]. Disturbing moments, acting on each crankshaft can be presented in the form of a Fourier series:

$$
\tilde{M}(t)=\sum_{i=1}^{N_{G}}\left(M_{i}^{(s)} \sin (i \Omega t)+M_{i}^{(c)} \cos (i \Omega t)\right),
$$

where $\Omega$ is the crankshaft rotational frequency. It is known that the third harmonic of the Fourier series is predominant and the most dangerous in this engine [16-18]. The disturbing moments acting on the crank can be approximated by taking only 


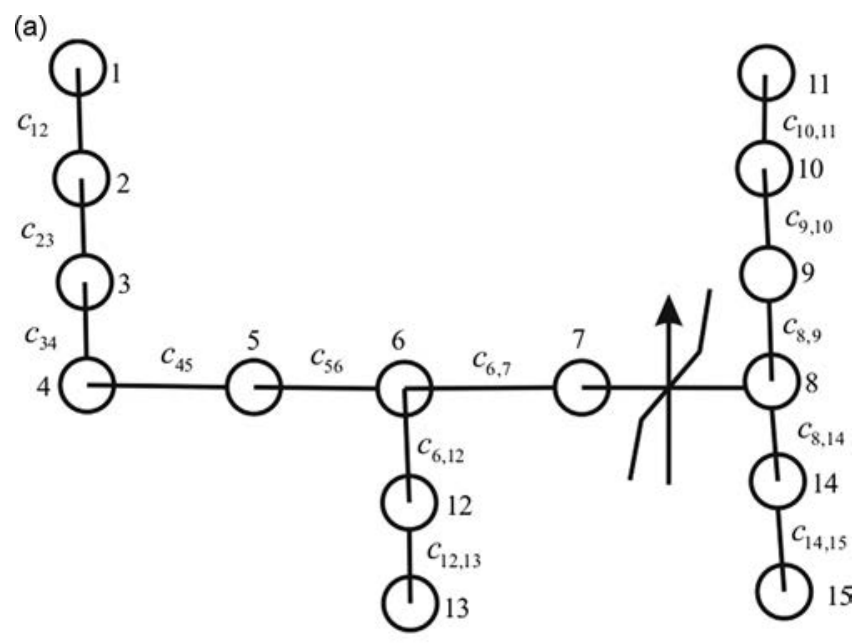

(b)

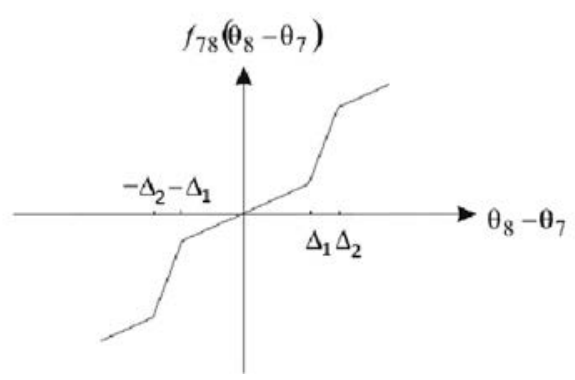

Fig. 3. Diagram of torsional vibrations.

the third harmonic of the Fourier series:

$$
\tilde{M}(t)=M_{3}^{(s)} \sin (3 \Omega t)+M_{3}^{(c)} \cos (3 \Omega t) .
$$

Methods for calculation of the disturbing moments $\tilde{M}(t)$ are treated in [20].

The moments $\tilde{M}(t)$ excite the torsional vibrations of the power transmission. As follows from an experimental analysis of the system [16-18] the vibrations of all system elements are approximated by one harmonic of the Fourier series $\sin (3 \Omega t+\vartheta)$. It is well known that the frequency response of the forced nonlinear vibrations outlines a backbone curve of free nonlinear vibrations [19]. In this paper the backbone curves are analyzed to study the system torsional vibrations, and nonlinear normal modes are used to obtain the backbone curves of the torsional vibrations.

A model of the torsional vibrations of the power transmission is considered. It consists of rigid disks and massless shafts and the approach from references [15,16] is used to obtain a finite degree of freedom model. Each crankshaft is described by three rigid disks and massless shafts attached to the disks. Such models of crankshaft torsional vibrations have been treated in [15]. Numerical values for the moments of inertia of the cranks and the shaft stiffnesses are obtained experimentally and by calculation. These experimental investigations and calculations have been published in reports $[17,18]$. The finite degree of freedom model of the torsional vibrations is shown in Fig. 3a. Disks 1, 2, 3 and 11, 10, 9 are used to approximate the crankshaft torsional vibrations. These discs are coupled by massless shafts with the following torsional stiffness $c_{12}, \quad c_{23}, c_{34}, c_{10,11}, c_{9,10}, c_{8,9}$ (Fig. 3a).

Now the model of the torsional vibrations of the gear train is considered. The gear train is shown in Fig. 1. The torsional vibrations of every gear are described by the vibrations of a rigid disk. These disks are coupled by massless shafts. These shafts describe the stiffnesses of the gears. The stiffnesses of two gears meshing are determined by the bending stiffness of a tooth and a tooth contact stiffness. The motions of the disks 4-8 (Fig. 3a) describe the torsional vibrations of the gears (Fig. 1). These disks are coupled by massless shafts with torsional stiffness $c_{4,5}, c_{5,6}, c_{6,7}$. The moments of inertia of these gears are calculated and the stiffnesses of two gears meshing are determined experimentally. The calculations of the moments of inertia and the results of the experimental analysis of these stiffnesses are treated in [17,18].

The disks 12 and 13 (Fig. 3a) describe the vibrations of a turbo-charge, and disk 13 describes the torsional vibrations of the turbine. The engine flywheel is denoted by disk 14. The power consumer is described by disk 15 .

The moment of inertia of rigid disk $i$ is denoted by $I_{i}$. The torsional vibrations of the disk $i$ are described by the generalized coordinate $\theta_{i}$. The stiffness of the shaft between the disks $i$ and $j$ is denoted by $c_{i j}$ (Fig. 3a). 
The clutch (Fig. 2) is described by the dependence of the torsional torque on the angle of torsion. This function is piecewise linear and it is shown in Fig. 3b. As follows from the experimental analysis of the torsional vibrations [17,18], the amplitudes of the system vibrations satisfy the following relation (Fig. 3b):

$$
-\Delta_{2}<\theta_{8}-\theta_{7}<\Delta_{2} \text {. }
$$

Therefore the nonlinear torsional torque of the clutch $f_{78}\left(\theta_{8}-\theta_{7}\right)$ is described by the following function:

$$
f_{78}\left(\theta_{8}-\theta_{7}\right)=\left\{\begin{array}{c}
c_{78}\left(\theta_{8}-\theta_{7}\right),-\Delta_{1} \leq\left(\theta_{8}-\theta_{7}\right) \leq \Delta_{1} \\
\tilde{c}_{78}\left(\theta_{8}-\theta_{7}\right)+\left(c_{78}-\tilde{c}_{78}\right) \Delta_{1}, \Delta_{1}<\left(\theta_{8}-\theta_{7}\right) ; \\
\tilde{c}_{78}\left(\theta_{8}-\theta_{7}\right)-\left(c_{78}-\tilde{c}_{78}\right) \Delta_{1},\left(\theta_{8}-\theta_{7}\right)<-\Delta_{1} .
\end{array}\right.
$$

The torsional vibrations within Fig. 3a are described by the following system of nonlinear ordinary differential equations:

$$
\begin{gathered}
I_{1} \ddot{\theta}_{1}-c_{12}\left(\theta_{2}-\theta_{1}\right)=0 ; \\
I_{i} \ddot{\theta}_{i}+c_{i-1, i}\left(\theta_{i}-\theta_{i-1}\right)-c_{i, i+1}\left(\theta_{i+1}-\theta_{i}\right)=0 ; i=2, \ldots, 5 ; \\
I_{6} \ddot{\theta}_{6}+c_{56}\left(\theta_{6}-\theta_{5}\right)-c_{67}\left(\theta_{7}-\theta_{6}\right)-c_{6,12}\left(\theta_{12}-\theta_{6}\right)=0 ; \\
I_{7} \ddot{\theta}_{7}+c_{67}\left(\theta_{7}-\theta_{6}\right)-f_{78}\left(\theta_{8}-\theta_{7}\right)=0 ; \\
I_{8} \ddot{\theta}_{8}+f_{78}\left(\theta_{8}-\theta_{7}\right)-c_{89}\left(\theta_{9}-\theta_{8}\right)-c_{6,14}\left(\theta_{14}-\theta_{8}\right)=0 ; \\
I_{i} \ddot{\theta}_{i}+c_{i-1, i}\left(\theta_{i}-\theta_{i-1}\right)-c_{i, i+1}\left(\theta_{i+1}-\theta_{i}\right)=0 ; i=9,10 ; \\
I_{11} \ddot{\theta}_{11}+c_{10,11}\left(\theta_{11}-\theta_{10}\right)=0 ; \\
I_{12} \ddot{\theta}_{12}+c_{6,12}\left(\theta_{12}-\theta_{6}\right)-c_{12,13}\left(\theta_{13}-\theta_{12}\right)=0 ; \\
I_{13} \ddot{\theta}_{13}+c_{12,13}\left(\theta_{13}-\theta_{12}\right)=0 ; \\
I_{14} \ddot{\theta}_{14}+c_{8,14}\left(\theta_{14}-\theta_{8}\right)-c_{14,15}\left(\theta_{15}-\theta_{14}\right)=0 ; \\
I_{15} \ddot{\theta}_{15}+c_{14,15}\left(\theta_{15}-\theta_{14}\right)=0 .
\end{gathered}
$$

The system (15) accepts the rigid body rotations with constant angular velocity. In order to eliminate this type of motions the following change of variables is used:

$$
\begin{gathered}
x_{1,2}=\theta_{2}-\theta_{1} ; x_{2,3}=\theta_{3}-\theta_{2} ; x_{3,4}=\theta_{4}-\theta_{3} ; x_{4,5}=\theta_{5}-\theta_{4} ; x_{5,6}=\theta_{6}-\theta_{5} ; \\
x_{6,7}=\theta_{7}-\theta_{6} ; x_{7,8}=\theta_{8}-\theta_{7} ; x_{8,9}=\theta_{8}-\theta_{9} ; x_{9,10}=\theta_{9}-\theta_{10} ; x_{10,11}=\theta_{10}-\theta_{11} ; \\
x_{8,14}=\theta_{8}-\theta_{14} ; x_{14,15}=\theta_{14}-\theta_{15} ; x_{6,12}=\theta_{6}-\theta_{12} ; x_{12,13}=\theta_{12}-\theta_{13} .
\end{gathered}
$$

The stiffnesses of the shafts are the following in $\mathrm{Nm}$ :

$$
\begin{gathered}
c_{12}=c_{23}=2 \times 10^{6} ; c_{34}=3 \times 10^{6} ; c_{45}=2.8 \times 10^{6} ; c_{56}=1.5 \times 10^{6} ; c_{67}=6.5 \times 10^{5} ; \\
c_{78}=8.4 \times 10^{5} ; \tilde{c}_{78}=5.04 \times 10^{5} ; c_{89}=2.6 \times 10^{6} ; c_{9,10}=c_{10,11}=2 \times 10^{6} ; \\
c_{6,12}=1.9 \times 10^{4} ; c_{12,13}=2.7 \times 10^{4} ; c_{8,14}=3.1 \times 10^{6} ; c_{14,15}=3.3 \times 10^{4} .
\end{gathered}
$$

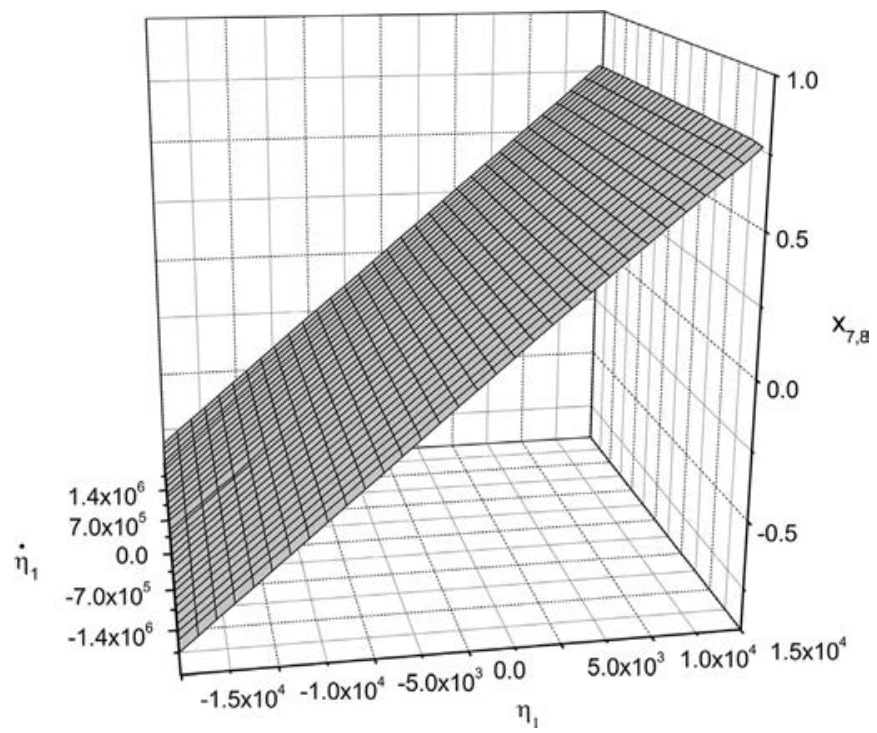

Fig. 4. Shaw-Pierre nonlinear normal modes in the coordinate frame $\left(\eta_{1}, \dot{\eta}_{1}, x_{7,8}\right)$. 
The numerical values of the disk moments of inertia are the following in $\mathrm{kg} \mathrm{m}^{2}$ :

$$
\begin{gathered}
I_{1}=I_{2}=I_{3}=7.7 \times 10^{-2} ; I_{4}=6.5 \times 10^{-2} ; I_{5}=8.5 \times 10^{-2} ; I_{6}=3.6 \times 10^{-2} ; \\
I_{7}=4.9 \times 10^{-2} ; I_{8}=3.8 \times 10^{-2} ; I_{9}=I_{10}=I_{11}=7.7 \times 10^{-2} ; I_{12}=1.7 ; \\
I_{13}=1 ; I_{14}=2.6 ; I_{15}=3 .
\end{gathered}
$$

The parameter $\Delta_{1}$ is taken with the following value: $\Delta_{1}=9.5 \times 10^{-2} \mathrm{rad}$.

Note that two stiffnesses of the clutch $c_{7,8}$ and $\tilde{c}_{7,8}$ (Fig. $3 \mathrm{~b}$ ) essentially differ, therefore, the dynamical system (15) is considered to be essentially nonlinear.

The Shaw- Pierre nonlinear normal modes are calculated next. The calculations are performed for the following range of the vibrations amplitudes: $a \in\left[0 ; 16 \Delta_{1}\right]$. This range is split into eight strips. The width of these strips is equal to $2 \Delta_{1}$. The amplitude boundary values of these strips are denoted by $a_{j}, a_{j+1}$. The following two trial functions $T_{l, m}(a, \varphi)$ are used for the expansion (9):

$$
T_{1,1}(a, \varphi)=\frac{a-a_{j}}{a_{j+1}-a_{j}} \cos \varphi ; T_{2,1}(a, \varphi)=\frac{a_{j+1}-a}{a_{j+1}-a_{j}} \cos \varphi
$$

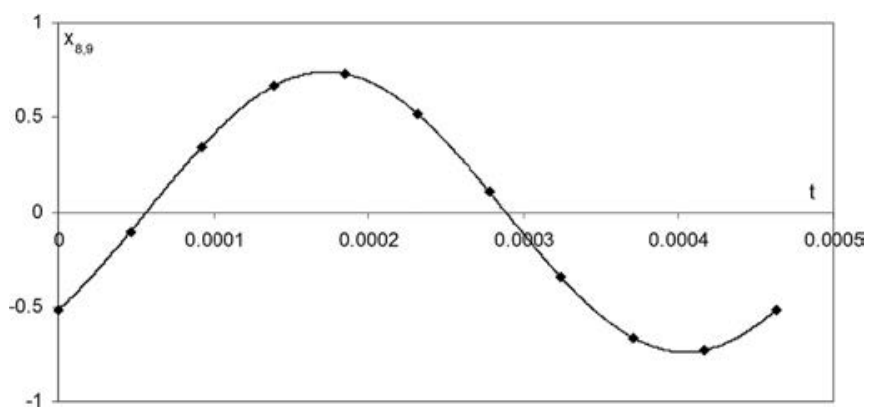

Fig. 5. System motion in time. Solid line shows the motions obtained from the nonlinear normal mode analysis. The points show the results of the direct numerical integration.

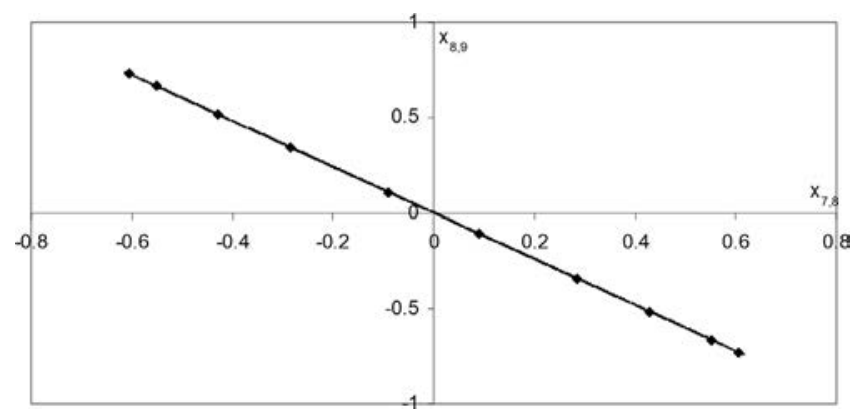

Fig. 6. The Kauderer-Rosenberg nonlinear normal mode in the configurational plane $\left(x_{7,8} ; x_{8,9}\right)$.

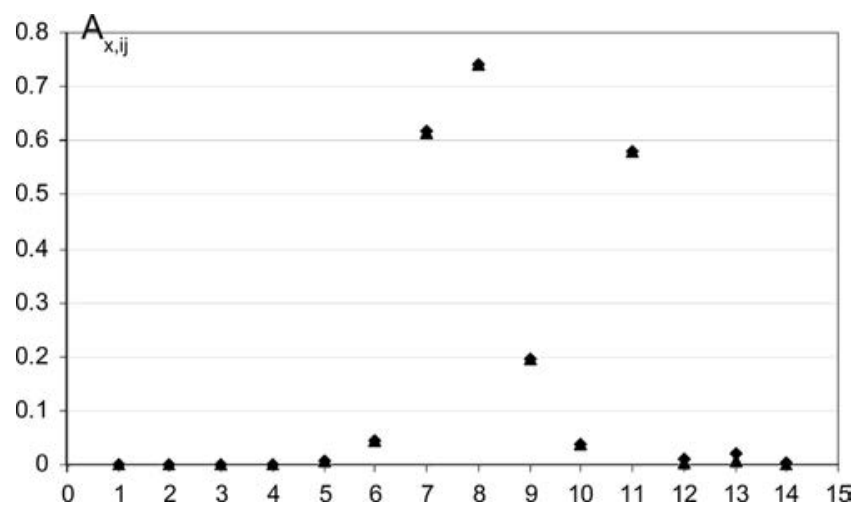

Fig. 7. Distribution of the vibrations amplitudes to the generalized coordinates $x_{i j}$. 


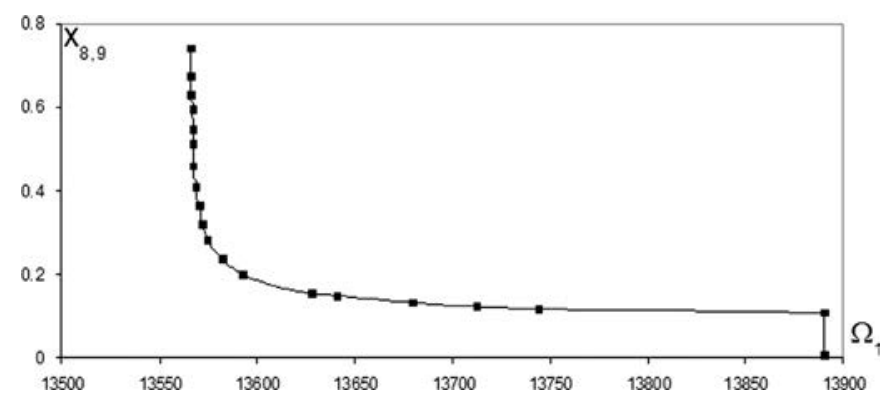

Fig. 8. Backbone curve of the generalized coordinate $x_{8,9}$.

(a)

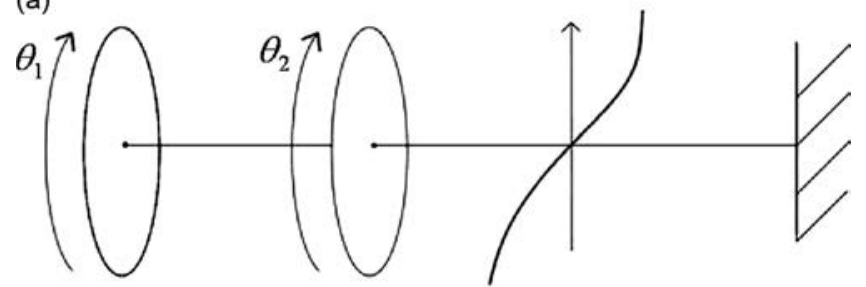

(b)

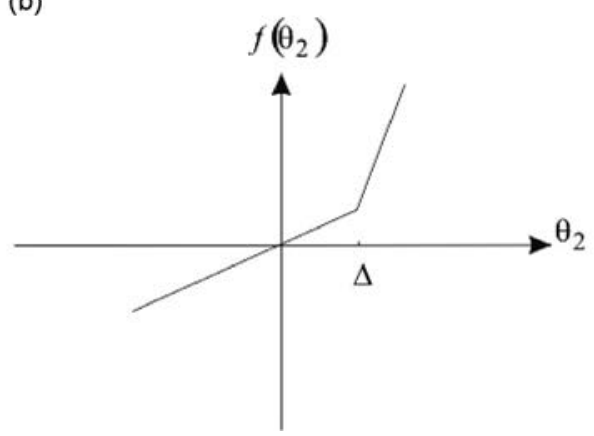

Fig. 9. Mechanical system (a) model of torsional vibrations and (b) elastic moment.

The results of the Shaw-Pierre nonlinear normal mode calculations in the coordinate frame $\left(\eta_{1}, \dot{\eta}_{1}, x_{7,8}\right)$ are shown in Fig. 4. Thus the obtained nonlinear normal mode is close to a plane.

A direct numerical integration of the dynamical system (15) from the initial conditions, which belong to the nonlinear normal mode (Fig. 4), is performed to verify the obtained nonlinear normal mode. The time dependence of the motions $x_{8,9}$ is shown in Fig. 5. The solid line shows the motions on the nonlinear normal mode (Fig. 4) and the points show the results of the direct numerical integration. Thus, the results are seen to be close.

The motions for the Shaw-Pierre nonlinear normal mode (Fig. 4) are presented in the form of the Kauderer-Rosenberg nonlinear normal mode. The motions in the configuration plane $\left(x_{7,8} ; x_{8,9}\right)$ are shown in Fig. 6 . The modal line is shown by the solid line. The results of the direct numerical integration are shown by points in Fig. 6 . The data of the direct numerical integration and the results of the nonlinear normal modes analysis are close.

Thus the periodic motions in the piecewise linear systems can be presented in the form of the Kauderer-Rosenberg nonlinear normal mode.

The distribution of the vibration amplitudes of the generalized coordinates on the nonlinear normal mode is analyzed, and the values of the vibration amplitudes versus the number of the generalized coordinates are shown in Fig. 7 . The number of the generalized coordinate is equal to the sequel number of the vector elements

$\left(x_{1,2} ; x_{2,3} ; x_{3,4} ; x_{4,5} ; x_{5,6} ; x_{6,7} ; x_{7,8} ; x_{8,9} ; x_{9,10} ; x_{10,11} ; x_{8,14} ; x_{14,15} ; x_{6,12} ; x_{12,13}\right)$.

As follows from Fig. 7, the generalized coordinates $x_{6,7}, x_{7,8}, x_{8,9}, x_{9,10}, x_{10,11}, x_{8,14}$ have significant vibration amplitudes on the nonlinear normal mode. The greatest amplitudes have the following motions $x_{7,8}, x_{8,9}, x_{8,14}$.

The nonlinear normal modes are calculated for different values of the vibrations amplitudes. The results of this analysis are shown by the backbone curve in Fig. 8. This figure shows the amplitude of $x_{8,9}$ versus the frequency $\Omega_{1}$. The direct numerical integration of the dynamical system (15) is carried out to verify the frequency response of Fig. 8, which is obtained by nonlinear normal modes numerical analysis. The results of the direct numerical integration are shown by points in Fig. 8. The data obtained by the two methods are clearly very close. 
Thus, nonlinear normal modes of the fifteen degrees of freedom system with one nonlinear element have been calculated. This system is very complex. The results of the calculations show the efficiency of the suggested method for nonlinear normal modes analysis.

\subsection{System with two degrees of freedom}

The torsional vibrations of a two degrees of freedom nonlinear dynamical system, which is shown in Fig. 9a, are considered. Note, that the properties of the nonlinear normal modes are analyzed by using this two degrees of freedom system. Periodic and chaotic motions of such systems have been treated by Avramov and co-authors [21-24]. The nonlinear elastic moment is shown in Fig. 9b. The mechanical system of Fig. 9a consists of two rigid disks, which have moments of inertia $I_{1}$ and $I_{2}$. These disks are coupled by an elastic shaft with stiffness $c_{1}$. An elastic shaft with a nonlinear element is located between the restraint and the disk. This nonlinear element is described by the piecewise linear function shown in Fig. 9b. The system motions are described by the following ordinary differential equations:

$$
\begin{gathered}
I_{1} \ddot{\theta}_{1}-c_{1}\left(\theta_{2}-\theta_{1}\right)=0 ; I_{2} \ddot{\theta}_{2}+c_{1}\left(\theta_{2}-\theta_{1}\right)+f\left(\theta_{2}\right)=0 ; \\
f\left(\theta_{2}\right)=\left\{\begin{array}{c}
c_{1} \theta_{2}, \theta_{2}<\Delta ; \\
c_{2} \theta_{2}-\Delta\left(c_{2}-c_{1}\right), \theta_{2} \geq \Delta,
\end{array}\right.
\end{gathered}
$$

where $\theta_{1}$ and $\theta_{2}$ are generalized coordinates.

(a)

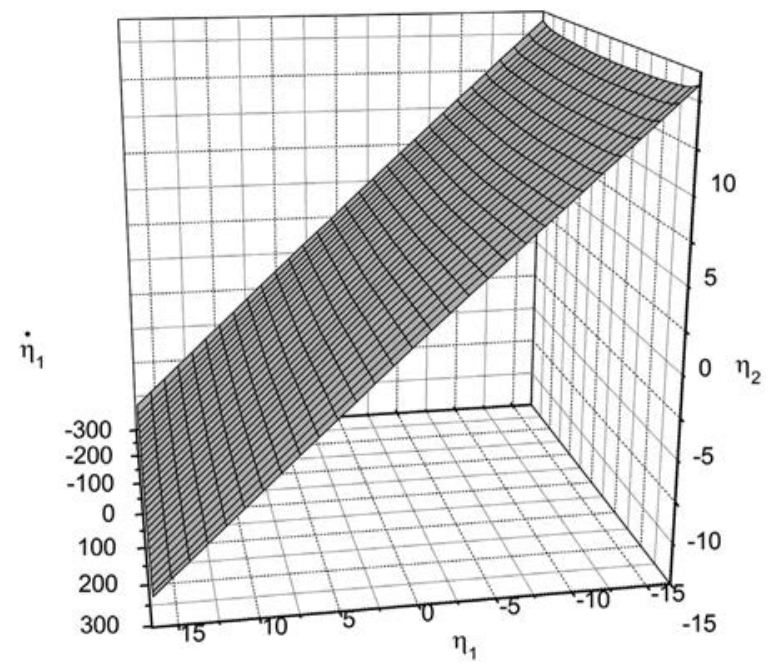

(b)

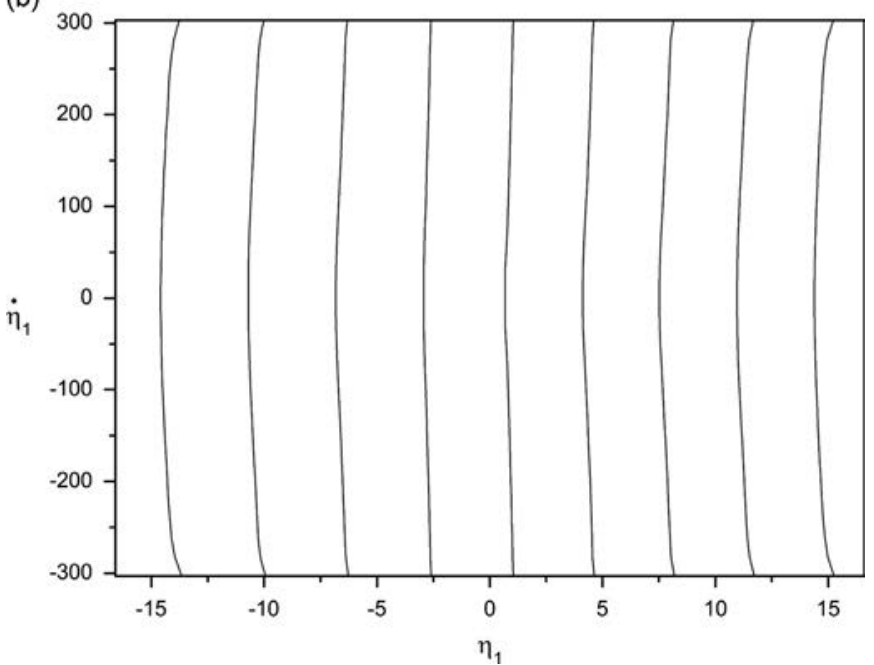

Fig. 10. Nonlinear mode for $c_{2}=0.5 c_{1}$; the surface in the coordinate frame $\left(\eta_{1}, \dot{\eta}_{1}, \eta_{2}\right)$ is shown in (a) and the level curves of this surface are shown in (b). 
The numerical values of the system parameters are as follows:

$$
c_{1}=150 \mathrm{~N} \mathrm{~m} ; \Delta=1.04 \mathrm{rad} ; I_{1}=0.3 \mathrm{~kg} \mathrm{~m}^{2} ; I_{2}=0.45 \mathrm{~kg} \mathrm{~m}^{2} .
$$

The eigenfrequencies of the system (17) for linear vibrations with small amplitudes are the following: $\omega_{1}=18.25 \mathrm{rad} / \mathrm{s}$; $\omega_{2}=44.72 \mathrm{rad} / \mathrm{s}$.

The nonlinear normal modes are calculated by using the approach suggested in Section 3. Fig. 10 shows the results of the calculations with the parameter $c_{2}=0.5 c_{1}$. Nonlinear normal mode is shown in the coordinate frame $\left(\eta_{1}, \dot{\eta}_{1}, \eta_{2}\right)$ in Fig. 10 a. As follows from this figure, the nonlinear normal mode is close to a plane. Level curves are calculated to distinguish between

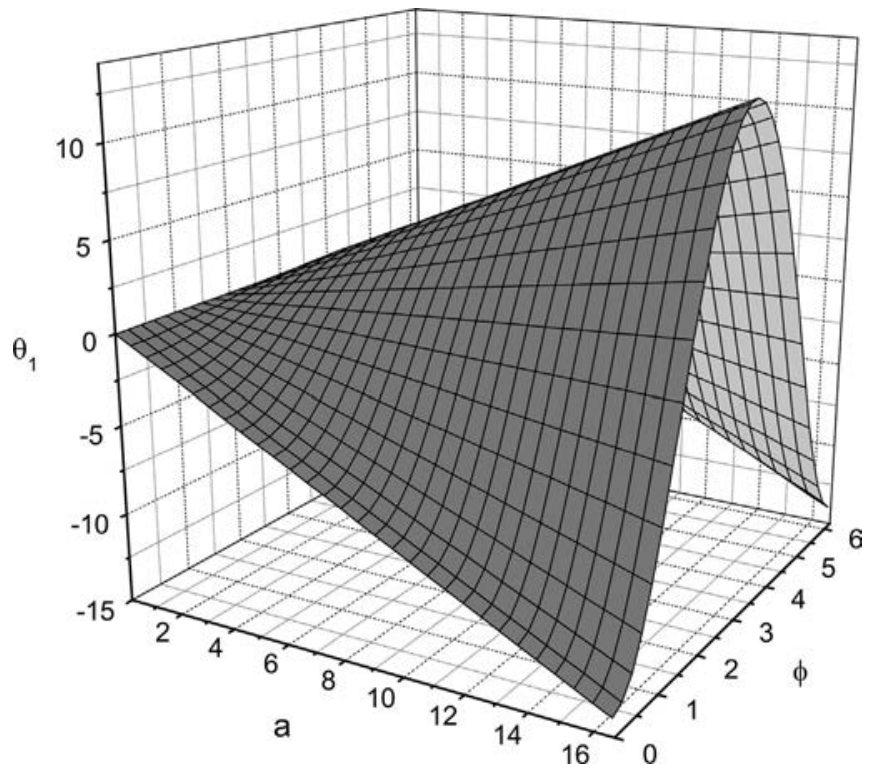

Fig. 11. The nonlinear normal mode in the coordinate frame $\left(a, \varphi, \theta_{1}\right)$.

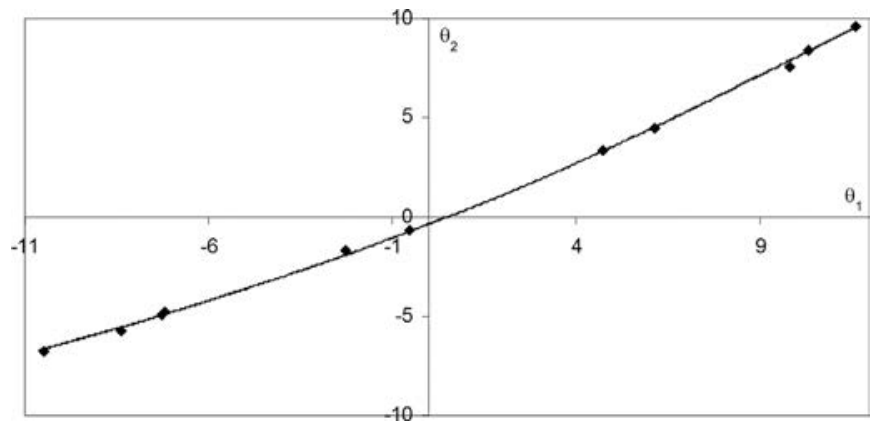

Fig. 12. Kauderer-Rosenberg nonlinear normal mode in configuration plane $\left(\theta_{1}, \theta_{2}\right)$.

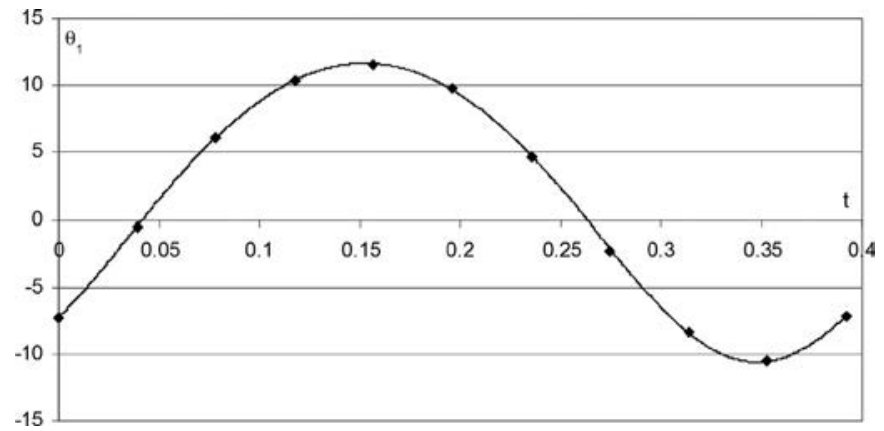

Fig. 13. The vibrations $\theta_{1}$ in time. 
the NNM from a plane. Fig. 10b shows these level curves. As follows from this figure the level curves differ from straight lines.

Fig. 11 shows this nonlinear normal mode in the coordinate frame $\left(a, \varphi, \theta_{1}\right)$. This coordinate frame is used in the papers $[8,9]$. The obtained nonlinear normal mode in Fig. 11 is reconstructed in the configurational plane $\left(\theta_{1}, \theta_{2}\right)$. The results are

(a)

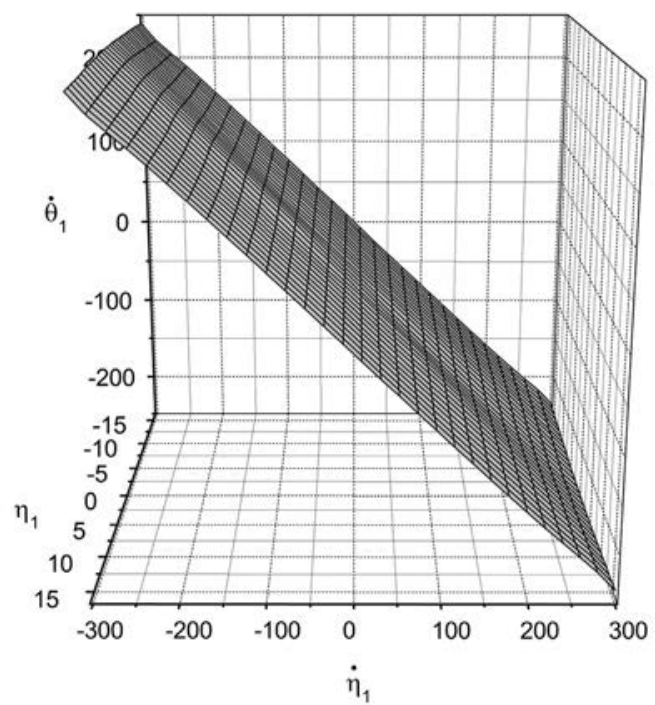

(b)

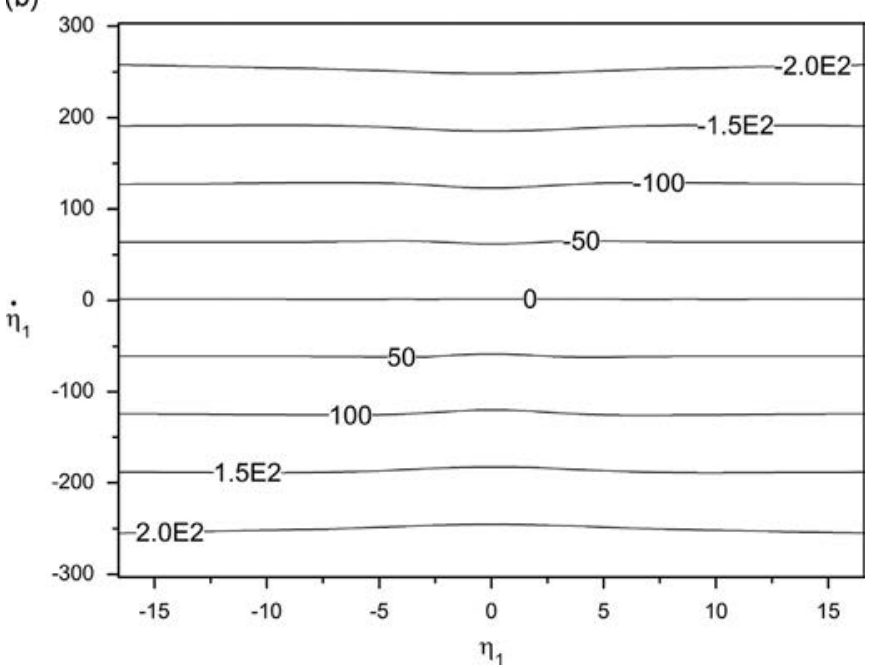

Fig. 14. Nonlinear mode. The surface in the coordinate frame $\left(\eta_{1}, \dot{\eta}_{1}, \dot{\theta}_{1}\right)$ is shown in (a) and level curves of this surface are shown in (b).

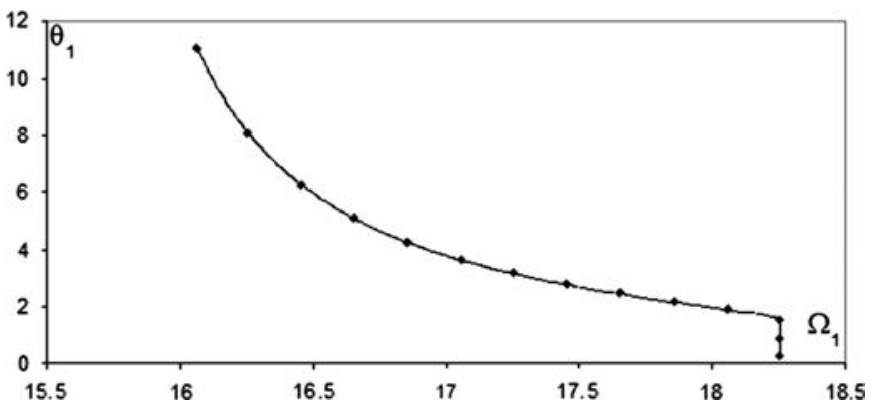

Fig. 15. Backbone curve of the vibrations $\theta_{1}$. 


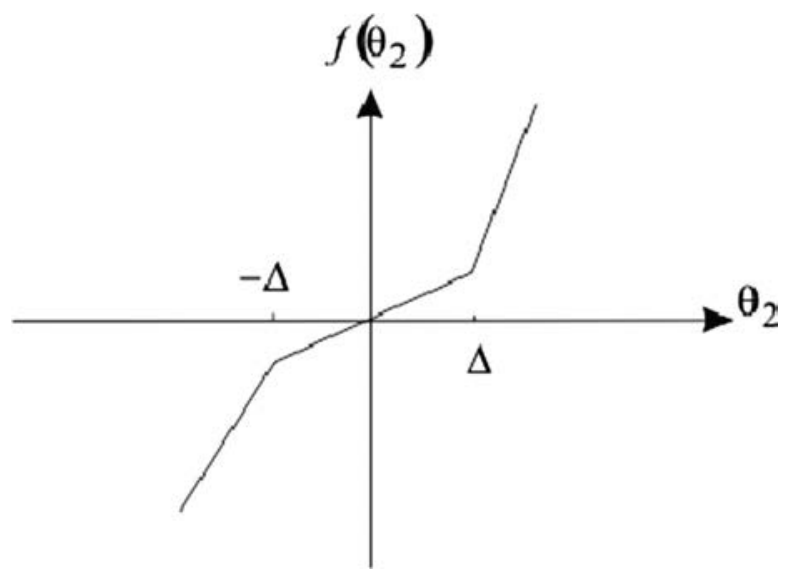

Fig. 16. The trilinear elastic moment.

(a)

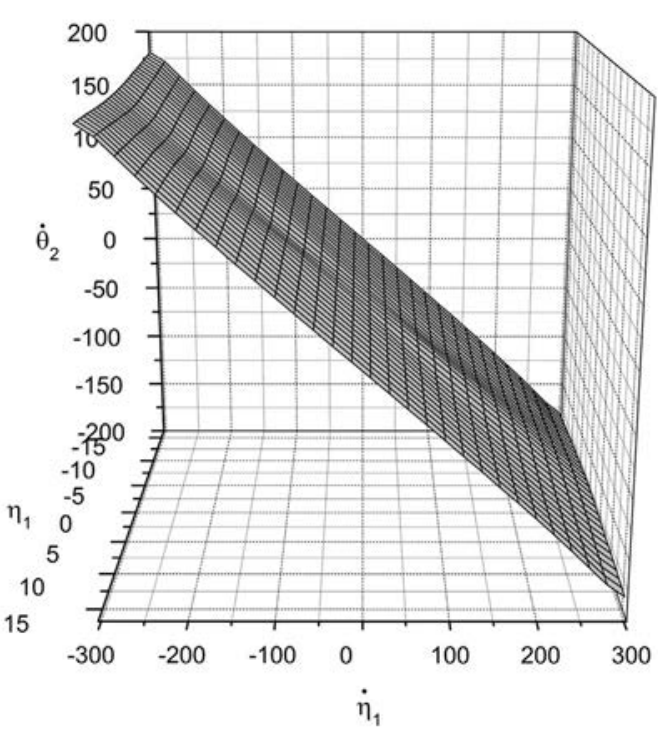

(b)

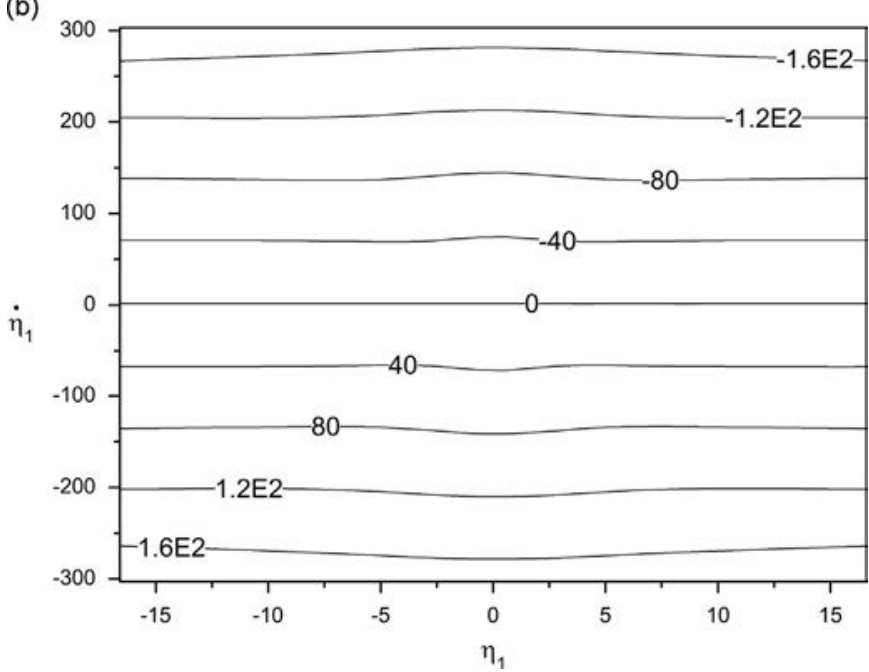

Fig. 17. Nonlinear normal mode of the system with trilinear elastic moment. The surface in the coordinate frame $\left(\eta_{1}, \dot{\eta}_{1}, \dot{\theta}_{2}\right)$ is shown in (a) and the level curves of this surface are shown in (b). 


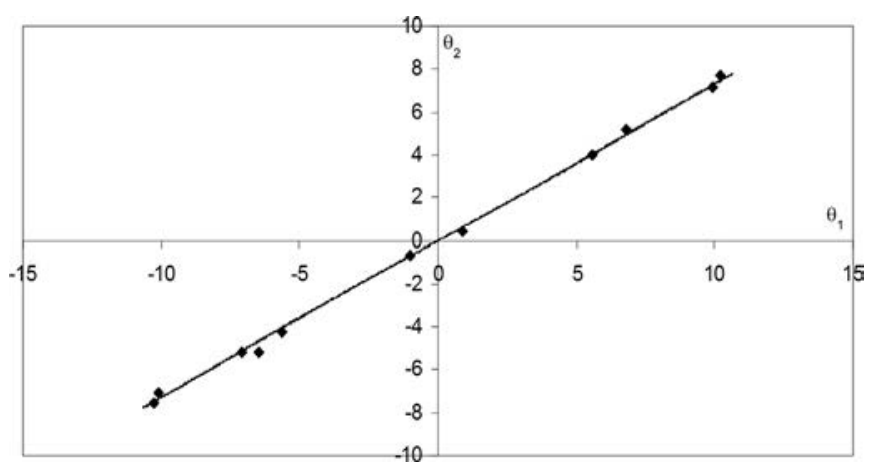

Fig. 18. Kauderer-Rosenberg nonlinear normal mode of the system with trilinear elastic force.

shown by the solid line on Fig. 12. Thus, the considered motions are Kauderer-Rosenberg nonlinear normal modes, as presented by the single-valued function $\theta_{2}\left(\theta_{1}\right)$.

Now several properties of the Kauderer-Rosenberg nonlinear normal mode are considered. In the first place, the nonlinear normal modes do not pass through the point $\theta_{1}=\theta_{2}=0$. In the second place, the nonlinear normal modes satisfy the following relation: $\left|\max \theta_{1}\right| \neq\left|\min \theta_{1}\right|$. These properties are explained by the asymmetry of the bilinear elastic force of the system (17) and (18).

To confirm the results of the nonlinear normal mode calculations, the direct numerical integration of the system (17) is carried out from the initial conditions, which belong to the nonlinear normal mode. The results of the calculations are shown by dots in Fig. 12. The data obtained by the two methods are close. The motion in time $\theta_{1}(t)$ on the nonlinear normal mode is shown by the solid line in Fig. 13 and the points indicate the results of the direct numerical integration.

As follows from Fig. 14, the nonlinear normal mode in the coordinate frame $\left(\eta_{1}, \dot{\eta}_{1}, \eta_{2}\right)$ of the bilinear system (17) and (18) is close to a plane. Fig. 14a shows this surface and the level curves are indicated in Fig. 14b.

The nonlinear normal mode is calculated for different values of the vibration amplitudes $a_{0}$. The frequencies of the vibrations $\Omega_{1}$ are determined for every value of the amplitude. The results of the calculations are shown by the backbone curve in Fig. 15, which is softening. The data of the direct numerical integration of the system (17) and (18) are shown by points in Fig. 15. The results of the nonlinear normal modes analysis and the data of the direct numerical integration are also seen to be close.

The torsional vibrations of the system (17) with the trilinear elastic moment are analyzed. The trilinear elastic moment has the following form:

$$
f\left(\theta_{2}\right)=\left\{\begin{array}{c}
c_{2} \theta_{2}+\Delta\left(c_{2}-c_{1}\right), \theta_{2} \leq-\Delta ; \\
c_{1} \theta_{2}, \quad-\Delta<\theta_{2}<\Delta ; \\
c_{2} \theta_{2}-\Delta\left(c_{2}-c_{1}\right), \theta_{2} \geq \Delta .
\end{array}\right.
$$

The graph of the nonlinear elastic moment (20) is shown in Fig. 16.

The nonlinear normal modes of the system (17) with the trilinear elastic moment for the parameters (19) and $c_{2}=0.7 c_{1}$ are analyzed. Fig. 17a shows the nonlinear normal mode in the coordinate frame $\left(\eta_{1}, \dot{\eta}_{1}, \dot{\theta}_{2}\right)$. The level curves of this nonlinear normal mode are shown in Fig. 17b. The same nonlinear normal mode in the configuration plane $\left(\theta_{1}, \theta_{2}\right)$ is shown in Fig. 18. Thus, the considered motions are Kauderer-Rosenberg nonlinear normal mode. This nonlinear normal mode $\theta_{2}\left(\theta_{1}\right)$ passes through the origin. This nonlinear normal mode is symmetrical with respect to the origin and this is the consequence of trilinear elastic moment symmetry.

The direct numerical integration of the system (15) from the initial conditions which belong to the nonlinear normal mode is carried out to verify the results of the nonlinear normal mode calculations. The results of the direct numerical integration are shown by points in Fig. 18. The closeness of the results obtained by the two methods confirms the correctness of the calculations.

\section{Conclusions}

It is proposed the approach for the Shaw-Pierre nonlinear normal modes calculations of piecewise linear finite degree of freedom systems is an alternative to the method suggested in $[9,10]$. The expansions for the slave generalized coordinates are used in the suggested method and the expansions for the slave generalized velocities are not considered. The Galerkin method is applied in order to obtain the system of nonlinear algebraic equations with respect to the coefficients of the nonlinear normal mode expansions. Due to the use of the suggested approach, the dimension of the nonlinear algebraic equations system for the nonlinear normal modes calculation is half as large as the dimension of the system suggested in $[16,21]$. 
The suggested method is used to analyze the nonlinear torsional vibrations of an internal combustion engine power transmission. The nonlinear models of these systems are discussed in $[16,17]$. We stress that models with many degrees of freedom are used to describe the vibrations of the internal combustion engine power transmission. Nonlinear normal modes are suitable for calculating the vibrations of such systems with many degrees of freedom.

As a result of the numerical calculations the following properties of the nonlinear normal modes are detected. It is shown that the nonlinear normal modes are close to a plane in the principal coordinates of the system linear region. These nonlinear normal modes differ essentially from a plane if they are presented in the coordinate system of both principal coordinates of the system linear region and the general velocity of the starting mechanical system.

As follows from the results of the numerical simulations the nonlinear normal modes of a piecewise linear system can be presented as modal lines in the configuration plane. Thus these motions are the Kauderer-Rosenberg nonlinear normal modes.

The distribution of the vibration amplitudes to the generalized coordinates on the nonlinear normal mode is very useful for detecting the most active generalized coordinates.

Level curves are very suitable for analyzing the properties of nonlinear normal modes.

\section{References}

[1] S.W. Shaw, P.J. Holmes, A periodically forced piecewise linear oscillator, Journal of Sound and Vibration 90 (1983) 129-155.

[2] R. Natsiavas, Stability and bifurcation analysis for oscillator with motion limiting constraints, Journal of Sound and Vibration 267 (1990) 97-102.

[3] G.H. Li, R.H. Rand, F.C. Moon, Bifurcation and chaos in a forced zero stiffness impact oscillator, International Journal of Non-Linear Mechanics 4 (1990) 417-432.

[4] R.S. Bishop, Impact oscillators, Philosophy Transactions of Royal Society A 347 (1994) 347-351.

[5] L.A. Ostrovsky, I.M. Starobinets, Transitions and statistical characteristics of vibrations in a bimodal oscillator, Chaos 5 (1995) 496-500.

[6] Y. Mikhlin, K.V. Avramov, Nonlinear normal modes for vibrating mechanical systems. Review of theoretical developments, Applied Mechanics Reviews 63 (2010) 4-20.

[7] S.C. Chen, S.W. Shaw, Normal modes for piecewise linear vibratory systems, Nonlinear Dynamics 10 (1996) 135-164.

[8] D. Jiang, C. Pierre, S.W. Shaw, Large-amplitude non-linear normal modes of piecewise linear systems, Journal of Sound and Vibration 272 (2004) 869-891.

[9] S.W. Shaw, C. Pierre, Normal modes for non-linear vibratory systems, Journal of Sound and Vibration 164 (1993) 85-124.

[10] E. Pesheck, C. Pierre, S.W. Shaw, A new Galerkin-based approach for accurate non-linear normal modes through invariant manifolds, Journal of Sound and Vibration 249 (2002) 971-993.

[11] K.V. Avramov, Yu.V. Mikhlin, Review of applications of nonlinear normal modes for vibrating mechanical systems, Applied Mechanical Reviewer 65 (2013) 20, http://dx.doi.org/10.1115/1.4023533.

[12] E. Pesheck, C. Pierre, S.W. Shaw, Accurate reduced-order models for a simple rotor blade model using nonlinear normal modes, Mathematical and Computer Modelling 33 (2001) 1085-1097.

[13] E. Pesheck, C. Pierre, S.W. Shaw, Modal reduction of a nonlinear rotating beams through nonlinear normal modes, ASME Journal of Vibration and Acoustics 124 (2002) 229-236.

[14] D. Jiang, C. Pierre, S.W. Shaw, The construction of non-linear normal modes for systems with internal resonances, The International Journal of Non-Linear Mechanic 40 (2005) 729-746.

[15] J.P. Den Hartog, Mechanical Vibrations, 4th ed. McGraw-Hill, New York, 1956.

[16] V.L. Veich, Ev. Kochura, Dynamics of Internal Combustion Engines, Machinostroenie, Sankt Petersburg, 1976 (in Russian).

[17] V.N. Karaban, K.V. Avramov, Dynamic Models and Free Vibrations of Power Transmission of Engine 3TD. Technical report, Kharkov, 2004 (in Russian).

[18] V.N. Karaban, K.V. Avramov, Damping of Torsional Vibrations of Three- cylinder Enginee. Forced Vibrations of Power Transmission of Engine 3TD. Technical report, Kharkov, 2004 (in Russian).

[19] A.H. Nayfeh, D.T. Mook, Nonlinear Oscillations, John Wiley and Sons, New York, 1979.

[20] P. Metallidis, S. Natsiavas, Linear and nonlinear dynamics of reciprocating engines, International Journal of Non-Linear Mechanics 38 (2003) $723-738$.

[21] K.V. Avramov, Yu. Mikhlin, Nonlinear Dynamics of Elastic Systems. Vol. 1. Models, Methods and Approaches, Scientific Centre "Regular and Chaotic Dynamics", Moscow, 2010 (in Russian).

[22] K.V. Avramov, Bifurcation analysis of a vibropercussion system by the method of amplitude surfaces, International Applied Mechanics 38 (2001) $1151-1156$.

[23] K.V. Avramov, V.N. Karaban, Resonance under random vibrations of discrete dynamic systems with piecewise-linear elastic characteristics, International Applied Mechanics 33 (1997) 584-588.

[24] K.V. Avramov, A.S. Belomyttsev, V.N. Karaban, Regions of chaotic oscillations of discrete mechanical systems with piecewise-linear elastic characteristics, International Applied Mechanics 30 (1994) 396-402. 